\title{
Circular RNA circPRMT5 is upregulated in breast cancer and is required for cell proliferation and migration
}

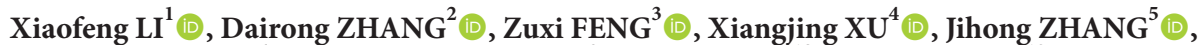

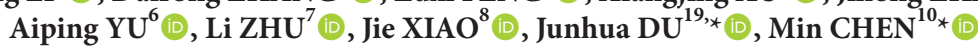 \\ ${ }^{1}$ Department of Public Health Management, Faculty of Medicine, Second People's Hospital of China Three Gorges University, Yichang, \\ China \\ ${ }^{2}$ Department of Urology Surgery, Faculty of Medicine, Second People's Hospital of China Three Gorges University, Yichang, China \\ ${ }^{3}$ Administrative Office, Second People's Hospital of China Three Gorges University, Yichang Second People's Hospital, Yichang, China \\ ${ }^{4}$ Department of Nursing, Faculty of Medicine, Second People's Hospital of China Three Gorges University, Yichang, China \\ ${ }^{5}$ Department of Integrated Traditional and Western Medicine, Faculty of Medicine, Second People's Hospital of China Three Gorges \\ University, Yichang, China \\ ${ }^{6}$ Department of Thyroid and Breast Tumor, Faculty of Medicine, Second People's Hospital of China Three Gorges University, Yichang, China \\ ${ }^{7}$ Department of Surgery, Faculty of Medicine, Second People's Hospital of China Three Gorges University, Yichang, China \\ ${ }^{8}$ Department of Medical Education, Faculty of Medicine, Second People's Hospital of China Three Gorges University, Yichang, China \\ ${ }^{9}$ Department of Tumor Chemoradiotherapy, Faculty of Medicine, Second People's Hospital of China Three Gorges University, Yichang, \\ China \\ ${ }^{10}$ Department of Nosocomial Infection, Faculty of Medicine, Second People's Hospital of China Three Gorges University, Yichang, China
}

Received: 07.02.2021 • Accepted/Published Online: 29.09.2021 • Final Version: 14.04.2022

Background/aim: To evaluate the role of cyclic protein arginine methyltransferase 5 (circPRMT5) in the occurrence and development of breast cancer (BC).

Materials and methods: A total of 90 BC patients who underwent radical mastectomy and 40 age-matched healthy female controls were recruited in the Second People's Hospital of China Three Gorges University, Yichang Second People's Hospital from 2017 to 2020. Quantitative reverse transcription polymerase chain reaction (RT-qPCR) was used to detect the expression levels of circPRMT5 in BC tissues, serum, normal breast cell line (MCF-10A), and BC cell line (T47D, MCF-7, BT549, Hs-578T, and MDA-MB-231, MDAMB-468). The associations between circPRMT5 expression level and age, tumor size, degree of differentiation, TNM stage, distant metastasis, estrogen receptor (ER) or progesterone receptor (PR), and human epidermal growth factor receptor 2 (HER2) status were analyzed. BC cell lines with circPRMT5 knockdown or overexpression were subject to CCK-8 cell proliferation assay, and transwell cell invasion/migration assay.

Results: CircPRMT5 expression in BC tissue was higher than that in adjacent normal breast tissue. Consistently, the expression level of circPRMT5 was also elevated in serum samples collected from BC patients when compared with healthy controls. And in multiple breast cancer cell lines, circPRMT5 was upregulated as compared to normal breast epithelial MCF-10A cells. CircPRMT5 expression level was correlated with tumor size, TNM stage, lymph node metastasis distant metastasis, but no correlation was observed with ER, PR, HER2 status. Overexpression of circPRMT5 promoted the proliferation, invasion, and migration of MCF7 cells; while the knockdown of circPRMT5 inhibited cell proliferation, invasion, and migration.

Conclusion: CircPRMT5 seems to act as an oncogene in the progression of BC. Our data suggest that CircPRMT5 may be used as a biomarker for the diagnosis, prognosis evaluation, and targeted therapy of breast cancer.

Key words: Breast cancer, circPRMT5, diagnosis, prognosis, proliferation, metastasis

\section{Introduction}

Breast cancer $(\mathrm{BC})$ is one of the malignant tumors with the highest incidence in women [1]. The annual incidence and death toll account for $23 \%$ and $14 \%$ of the global diagnosis and deaths respectively, ranking as one of the

\footnotetext{
*Correspondence: 1350655724@qq.com
}

leading causes of cancer-related fatality in females [2]. At present, there is no good biomarker for early diagnosis and prognostic evaluation of BC. Uncontrolled proliferation, invasion, and metastasis are important biological characteristics of $\mathrm{BC}$ cells, accounting for the recurrence 
and poor prognosis after initial treatment [3]. Distant metastasis has been considered one of the main causes of BC-related death [4]. Bone metastasis occurs in $70 \%$ of BC cases, which often correlates with a poor prognosis [5]. Therefore, the investigation of the molecular mechanism of $\mathrm{BC}$ growth and metastasis will provide new insight into novel biomarkers and the development of targeted therapy for effective $\mathrm{BC}$ diagnosis and treatment.

Circular RNA (circRNA) is a class of noncoding RNA molecule with a closed loop structure. The mechanism of circRNA molecule formation mainly involves "exon skipping" and "reverse shearing" of precursor mRNA [6]. Early studies proposed that circRNA molecules are redundant and nonfunctional molecules [7]. In recent years, the advancement of the deep sequencing technology enables the identification of a large number of exon or intron of circRNA, indicating that circRNAs are functional RNA molecules with regulatory properties [8,9]. Furthermore, the unique stable closed-loop structure of circRNA makes it a promising marker for clinical diagnosis. Indeed, several studies have revealed that circRNA molecules can be detected in the plasma of cancer patients, which are closely related to the survival of the patients [10-12].

CircPRMT5 (hsa_circ_0031242) is a circRNA implicated in the occurrence and development of a variety of malignant tumors [13, 14]. CircPRMT5 has been found to be highly expressed in hepatocellular carcinoma, bladder cancer, and nonsmall cell lung cancer, and it acts as an oncogene $[15,16]$. However, the role and the regulatory mechanism of circPRMT5 in $\mathrm{BC}$ remain to be uncovered. In this study, we collected BC tumor samples and adjacent normal tissues, as well as the serum samples from $\mathrm{BC}$ patients and healthy controls. We evaluated the expression level of circPRMT5 in BC tumor tissues and the serum sample of $B C$ patients. We further investigated the functional role of circPRMT5 overexpression in BC cells.

\section{Materials and methods}

\subsection{Patients and tissue samples}

This study was approved by the Institutional Review Board of Second People's Hospital of China Three Gorges University, Yichang Second People's Hospital (LL-2016011). Patient samples were obtained after written informed consent had been obtained, and all procedures were performed in this study involving human participants were in accordance with the Declaration of Helsinki (as revised in 2013). We collected BC tumor tissues and normal tissue adjacent to the tumor (paracancer tissues) from 90 patients during surgical resection at the Second People's Hospital of China Three Gorges University, Yichang Second People's Hospital between 2017 and 2020 (age range 18-80 years). Specimens were immediately frozen in liquid nitrogen after resection and stored at $-80{ }^{\circ} \mathrm{C}$ until usage. Each sample (5 g) was used for total RNA extraction and the expression of circPRMT5 in these samples was compared by RT-qPCR.

Inclusion criteria: Subjects who were able to provide written informed consent to participate in the study on a voluntary basis; subjects had no other concurrent malignant tumors; subjects with primary tumors that could be surgically removed, no blood-related diseases and infectious diseases were observed. Exclusion criteria: Subjects who were undergoing chemotherapy; subjects had fatal diseases; subjects with benign tumors, and patients with nonprimary breast lesions. All collected tumor tissues were diagnosed by three pathologists. The tumor site was confirmed by hematoxylin-eosin (H\&E) staining, and then the tumor and paracancer tissues were separated. H\&E staining was performed for a second time to further confirm the successful separation of tumor and nontumor tissues (see the flowchart in Figure 1). The pathological diagnosis of breast cancer was performed according to the WHO Classification (https://radiopaedia. org/articles/who-classification-of-tumors-of-the-breast) and the Elston and Ellis grading system (http://tvmouse. ucdavis.edu/bcancercd/311/grading_diagram.html).

Additionally, 2 weeks before the surgical operation, 40 out of $90 \mathrm{BC}$ patients were randomly selected and the circPRMT5 expression level in serum was compared to that of 40 age-matched healthy female volunteers enrolled in the same hospital (see the flowchart in Figure 1). Peripheral venous blood samples were collected 2 weeks before the surgical operation in the $\mathrm{BC}$ patients to detect the expression of circPRMT5 in serum. All the 90 BC patients were followed up regularly by telephone every month after surgery, and the recurrence and survival status of patients were recorded. Relapse-free survival time was defined as the time interval from the date of surgery to the date of the first clinical diagnosis of recurrence, and censored data was defined as the time interval from the date of surgery to the date of the last follow-up of the patients.

\subsection{Cell culture and transfection}

Human BC cell lines (T47D, MCF-7, BT549, Hs-578T and MDA-MB-231, MDA-MB-468) and normal breast epithelial MCF-10A cells were obtained from the Cell Bank of Chinese Academy of Sciences (Shanghai, China). Cells were cultured in RPMI-1640 medium (Gibco, USA) supplemented with $10 \%$ fetal bovine serum and $1 \%$ penicillin/streptomycin at $37^{\circ} \mathrm{C}$ in a humidified cell incubator with $5 \% \mathrm{CO}_{2}$. Cell transfection procedure was performed as described below:

MCF7 cells were trypsinized and centrifuged at $500 \times$ $\mathrm{g}$ for $5 \mathrm{~min}$, and then resuspended in complete medium. Cells were seeded into a 6 -well plate at a density of $5 \times$ $10^{5}$ cells/well and incubated overnight. When the cell density reached $60 \%$ to $80 \%$ confluency, transfection 


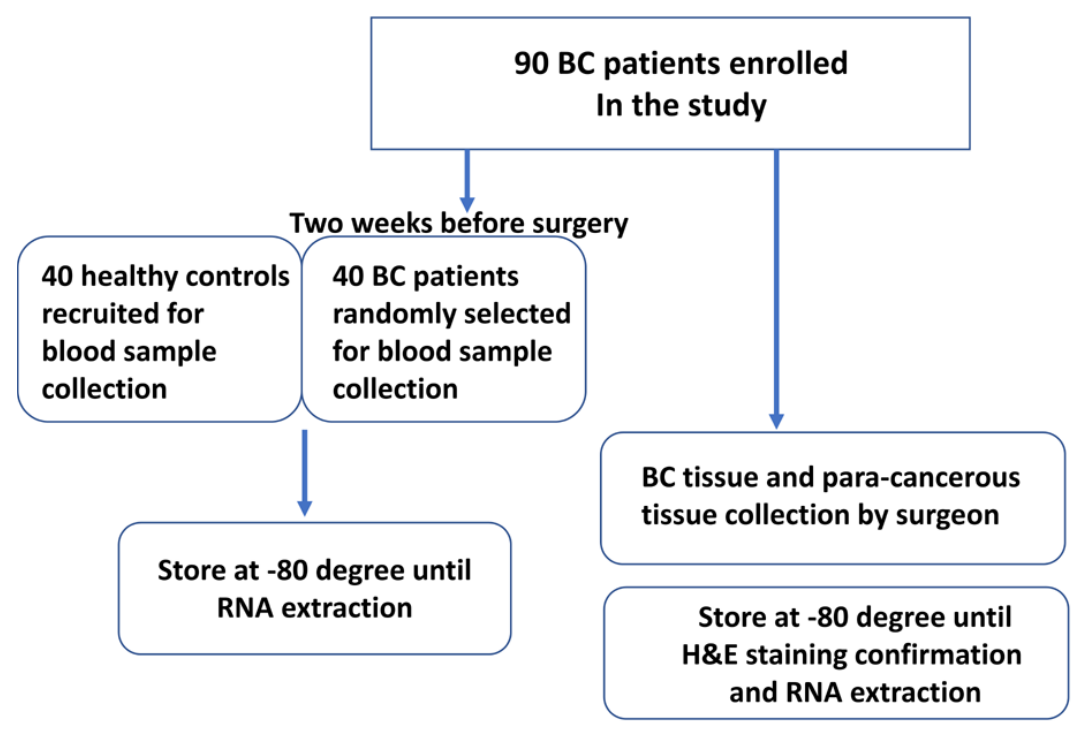

Figure 1. Flow diagram showing patient enrollment and clinical sample collection.

of siRNA (small interfering RNA) was conducted using Lipofectamine ${ }^{\mathrm{mt}}$ RNAiMAX transfection reagent (Invitrogen, USA) according to the manufacturer's manual. Briefly, $4 \mu \mathrm{L}$ of nonspecific siRNA (si-NC) or siRNA targeting circPRMT5 (si-circPRMT5) (100 $\mathrm{nM}$ ) were diluted with $200 \mu \mathrm{L}$ of opti-MEM medium (Thermo Fisher Scientific, USA), respectively. Then 8 $\mu \mathrm{L}$ of RNAiMAX transfection reagent was mixed with $200 \mu \mathrm{L}$ of opti-MEM medium. The diluted siRNA was mixed with transfection reagent at room temperature for 15 min, and then added to MCF7 cells. After $6 \mathrm{~h}$, cells were replenished with fresh medium for $36 \mathrm{~h}$ incubation before subsequent experiments. siRNA targeting circPRMT5 and siRNA control were purchased from Genscript Biotech (Nanjing, China). The oligonucleotide sequences are as follows: si-circPRMT5 sense chain: 5'-CUCCUGACCUCAGUUCAUCTT-3', antisense strand: 5'-GAUGAACUGAGGUCAGGAGTT-3'.

\subsection{Overexpression of circPRMT5}

The control vector and circPRMT5 overexpression plasmid pCDNA3.1-circPRMT5 were purchased from Genscript Biotech (Nanjing, China). The day before transfection, MCF7 cells were seeded in a 6-well plate at a density of $6 \times 10^{5}$ cells per well to ensure that the cells reached $70 \%$ confluence before transfection. Lipofectamine 2000 reagent $(5 \mu \mathrm{L})$ (Thermo Fisher Scientific, USA) was diluted with $125 \mu \mathrm{L}$ of opti-MEM medium. Plasmid $(2.5 \mu \mathrm{g})$ was diluted with $125 \mu \mathrm{L}$ of opti-MEM medium. The diluted plasmid was mixed with Lipofectamine 2000 reagent (1:1) and incubated for $15 \mathrm{~min}$ at room temperature. The mixed plasmid liposome complex was added to the cell culture. After $6 \mathrm{~h}$, fresh medium was replaced and cells were further cultured for $36 \mathrm{~h}$ before following experiments.

\subsection{RNA isolation and RT- $\mathrm{qPCR}$}

Total RNA was isolated from serum, tissue, and BC cell lines using a Trizol reagent (Takara Biotechnology Co., Ltd., Dalian, China) according to the manufacturer's instructions. The purity of the extracted RNA was determined by spectrophotometry at 260/280 nm, and $5 \mu \mathrm{g}$ purified total RNA was reverse transcribed into cDNA using the PrimeScript RT reagent kit (Cat\# RR037A, Takara, China). Quantitative real-time PCR (qPCR) reactions were performed on ABI Prism 7500 real time PCR instrument (Applied Biosystems, USA) using the SYBR Green PCR Master Mix (Takara, Japan). The PCR conditions were as follows: one cycle of $50^{\circ} \mathrm{C}$ for $2 \mathrm{~min}$ and $95^{\circ} \mathrm{C}$ for $10 \mathrm{~min}$, followed by 40 cycles of $95^{\circ} \mathrm{C}$ for $30 \mathrm{~s}$ and $60^{\circ} \mathrm{C}$ for $30 \mathrm{~s}$. The relative expression of the target gene was determined by $2-\Delta \Delta \mathrm{Ct}$ method. GAPDH was selected as the internal control gene for normalizing the expression. The primer sequences used for RT-qPCR were listed below [16]: GAPDH forward primer 5'- TCCCATCACCATCTTCCAGG -3', reverse primer 5'-GATGACCCTTTTGGCTCCC-3'; circPRMT5 forward primer 5'- TACCATTGGCCTCTAGCCCT-3', reverse primer 5'- CAAGGGGAATCACAGCCCAT-3'.

2.5. CCK-8 (cell counting kit-8) assay for cell proliferation CCK-8 cell proliferation assay was performed according to a previous study [17]. Cell counting kit-8 (CCK-8; Dojindo, Japan) assay was used for the cell proliferation capacity. Cells were seeded in a 96-well plate at a density of 3000 cells/well, and cultured for indicated time period. CCK-8 solution $(10 \mu \mathrm{L})$ was added to the cell culture at each time point, the plates were incubated for $3 \mathrm{~h}$. CCK- 8 $(10 \mu \mathrm{L})$ reagent was added to each well at the indicated time point and incubated in the incubator for $1 \mathrm{~h}$. A 
A

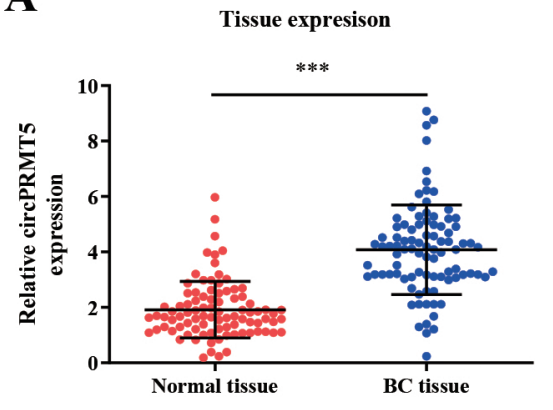

C

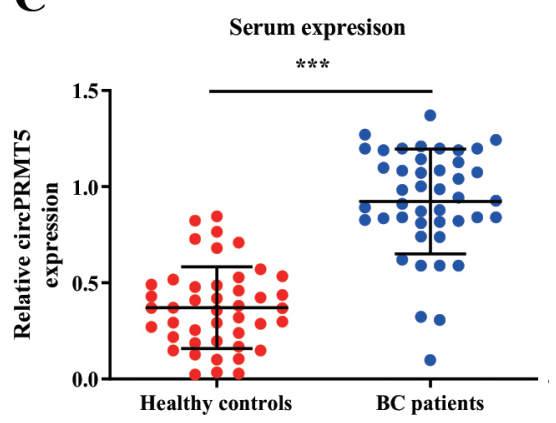

B

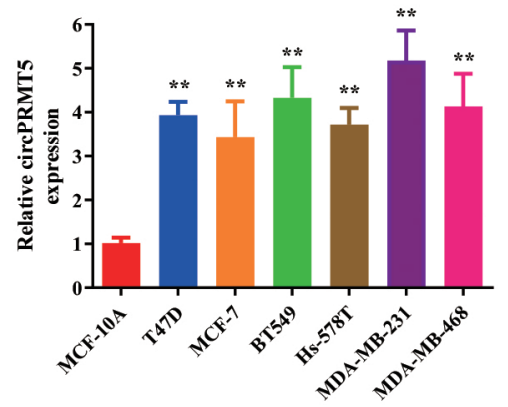

D

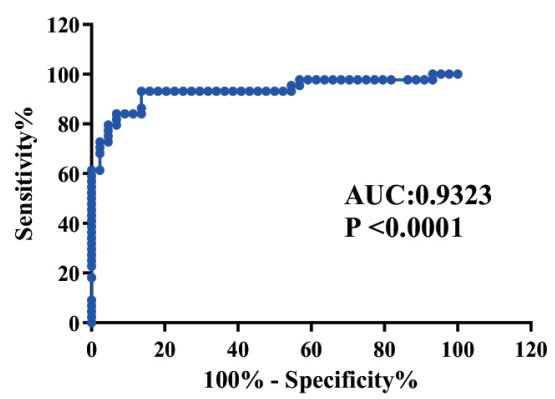

Figure 2: Expression of circPRMT5 in BC. A: the RT-qPCR method detects the expression level of circPRMT5 in 90 pairs of BC tumor tissues and adjacent normal tissues (normal, paracancer tissues). CircPRMT5 expression was significantly increased in BC tumor samples, $\mathrm{p}<0.001$. B: RT-qPCR method was used to measure the expression level of circPRMT5 in BC cell lines (T47D, MCF-7, BT549, Hs-578T, MDA-MB-231, and MDA-MB-468) and normal breast epithelial MCF-10A cells. The expression of circPRMT5 in BC cell lines were significantly elevated, $\mathrm{p}<0.01$. C: RT-qPCR method was used to detect the expression level of circPRMT5 in the serum samples of 40 cases of BC patients and 40 normal healthy controls. CircPRMT5 expression in BC serum was significantly increased, $\mathrm{p}<0.001$. D: Receiver operating characteristics (ROC) curve of circPRMT5 expression level for predicting the BC cancer from the 90 BC tumor samples and 90 paracancerous tissues. AUC (area under the curve) $>0.8$ indicates a good prediction with $\mathrm{p}<0.001$.

microplate reader (Bio-Rad, CA, USA) was used to detect the absorbance value (OD value) in each well at $450 \mathrm{~nm}$.

\subsection{Cell migration and invasion assays}

Cell migration and invasion assays were performed according to a previous study [18]. Corning Transwell polycarbonate membrane cell culture inserts with $8.0-\mu \mathrm{m}$ pores were uncoated for migration assay, or coated with $50 \mu \mathrm{L}$ of BD Matrigel Basement Membrane Matrix (BD Biosciences, USA) diluted 1:3 with FBS-free DMEM (fetal bovine serum free Dulbecco's Modified Eagle Medium) for invasion assay. Cells were trypsinized and then resuspended in serum-free medium. $2 \times 10^{4}$ cells were seeded in the top chamber with serum-free medium and allowed to migrate toward serum-containing medium in the lower chamber for $24 \mathrm{~h}$. Then cells were fixed in $4 \%$ paraformaldehyde and stained with $0.01 \%$ crystal violet (AS1086, ASPEN, China) for $10 \mathrm{~min}$. After wiping the cells remaining in the upper chamber with cotton swabs, the migratory or invasive cells were imaged and counted using a Leica AM6000 microscope (Leica Microsystems, Germany). The numbers of migratory or invasive cells were calculated as the average numbers of five random fields per well under 100X magnification.

\subsection{Statistical analysis}

Statistical analyses were performed with SPSS 20.0 software (IBM SPSS, Armonk, NY, USA). All the experiments were repeated three times. CircPRMT5 expression in tissues was examined in 90 pairs of cancer and paracancerous normal tissues; circPRMT5 expression in serum was analyzed from the blood samples of $40 \mathrm{BC}$ patients and 40 healthy controls. The association between circPRMT5 expression in cancer tissues and the clinic pathological parameters was evaluated with chi-square analysis. The statistical difference between the two groups was compared using unpaired student's t-tests. Comparisons among multiple groups were analyzed using one-way analysis of variance (ANOVA) with Tukey's post hoc test for pairwise comparison. Comparisons of data at multiple time 
A

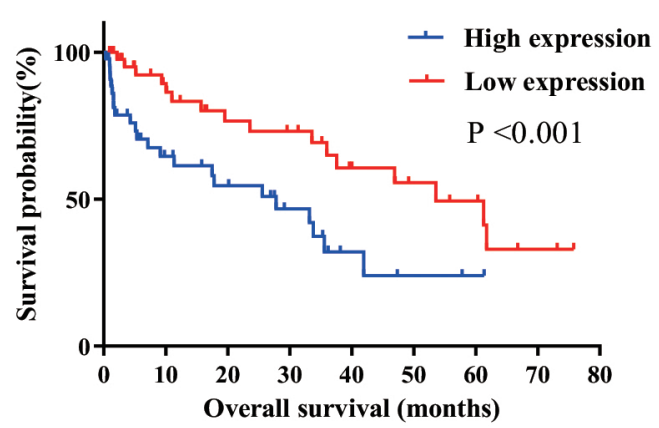

B

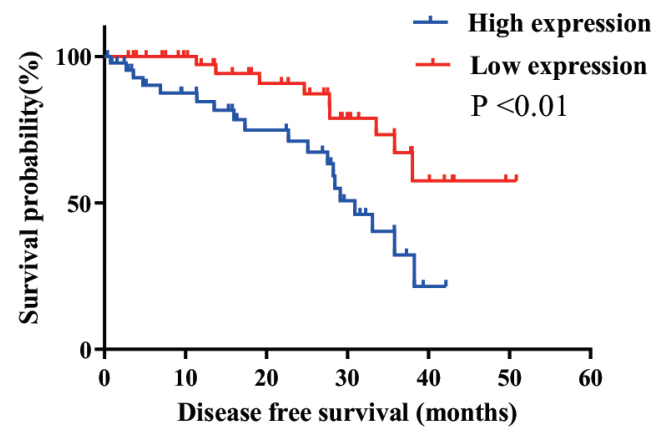

Figure 3. Relationship between circPRMT5 and prognosis of BC patients. A: Kaplan-Meier survival curve was used to assess the overall survival of BC patients in circPRMT5 low expression group $(n=45)$ and circPRMT5 high expression group $(n=45) .90$ patients were divided into low and high expression group based on the median value of circPRMT5 expression detect by qPCR in Figure 1A. BC patients in the circPRMT5 high expression group showed a poorer survival than that of low expression group, p $<0.001$. B: KaplanMeier survival curve was used to evaluate the disease-free survival time of BC patients in circPRMT5 low expression group ( $\mathrm{n}=45$ ) and the circPRMT5 high expression group $(\mathrm{n}=45)$. BC patients in the circPRMT5 high expression group had worse progression-free survival than that of low expression group, $\mathrm{p}<0.01$.

Table. Correlations of CircPRMT5 expression with clinicopathologic features of breast cancer.

\begin{tabular}{|c|c|c|c|c|c|}
\hline \multirow{2}{*}{\multicolumn{2}{|c|}{ Parameters }} & \multirow{3}{*}{$\begin{array}{l}\text { Total } \\
38\end{array}$} & \multirow{2}{*}{\multicolumn{2}{|c|}{$\begin{array}{l}\text { CircPRMT5 } \\
\text { Low/high }\end{array}$}} & \multirow{4}{*}{\begin{tabular}{|l} 
P-value \\
0.088
\end{tabular}} \\
\hline & & & & & \\
\hline \multirow{2}{*}{ Age } & $>50$ & & 15 & 23 & \\
\hline & $\leq 50$ & 52 & 30 & 22 & \\
\hline \multirow{2}{*}{ Tumor size } & $>2 \mathrm{~cm}$ & 34 & 25 & 9 & \multirow{2}{*}{0.001} \\
\hline & $\leq 2 \mathrm{~cm}$ & 56 & 20 & 36 & \\
\hline \multirow{2}{*}{ Differentiation } & High (well) & 36 & 28 & 8 & \multirow{2}{*}{0.001} \\
\hline & Low (poor) & 54 & 17 & 37 & \\
\hline \multirow{2}{*}{ TNM stage } & $\mathrm{I}-\mathrm{II}$ & 39 & 28 & 11 & \multirow{2}{*}{0.001} \\
\hline & III-IV & 51 & 17 & 34 & \\
\hline \multirow{2}{*}{ Lymph node metastasis } & Negative & 43 & 31 & 12 & \multirow{2}{*}{0.001} \\
\hline & Positive & 47 & 14 & 33 & \\
\hline \multirow{2}{*}{ Distant metastasis } & M0 & 58 & 39 & 19 & \multirow{2}{*}{0.001} \\
\hline & M1 & 32 & 6 & 26 & \\
\hline \multirow{2}{*}{ ER status } & Positive & 28 & 15 & 13 & \multirow{2}{*}{0.649} \\
\hline & Negative & 62 & 30 & 32 & \\
\hline \multirow{2}{*}{ PR status } & Positive & 26 & 9 & 17 & \multirow{2}{*}{0.063} \\
\hline & Negative & 64 & 36 & 28 & \\
\hline \multirow{2}{*}{ HER-2 status } & Positive & 26 & 11 & 15 & \multirow{2}{*}{0.352} \\
\hline & Negative & 64 & 34 & 30 & \\
\hline
\end{tabular}

points were examined using two-way ANOVA. Kaplan Meier Curve and log-rank test were used to compare the cumulative survival rates in $90 \mathrm{BC}$ patients. Data were reported as the mean \pm standard deviation $(\mathrm{SD}) . \mathrm{P}<0.05$ was considered to be statistically different.

\section{Results}

\subsection{Expression of circPRMT5 in BC}

To evaluate circPRMT5 expression in BC tumor samples, we used RT-qPCR to compare the expression level of circPRMT5 in 90 pairs of BC tissues and paired 
A
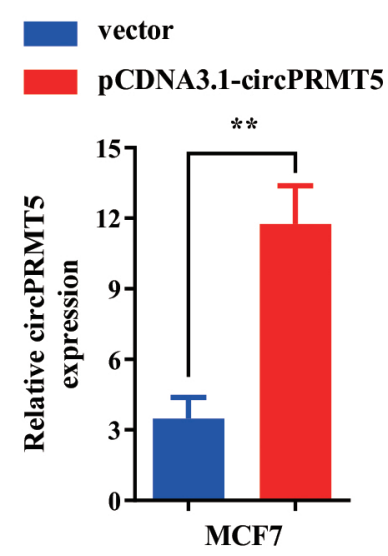

C

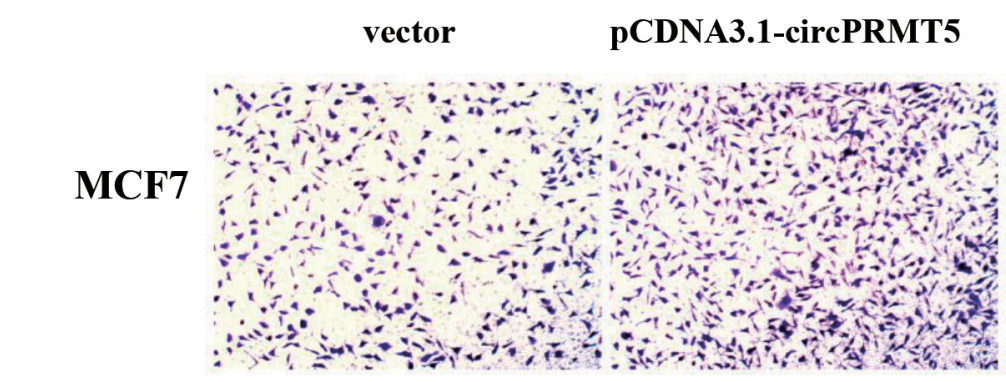

D

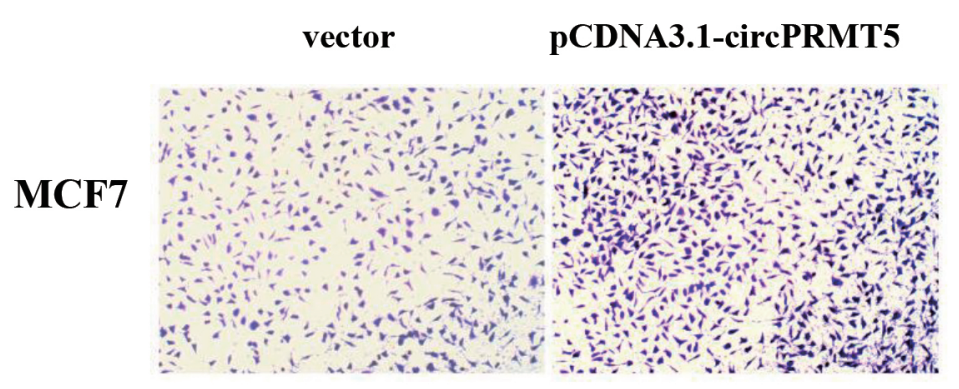

B

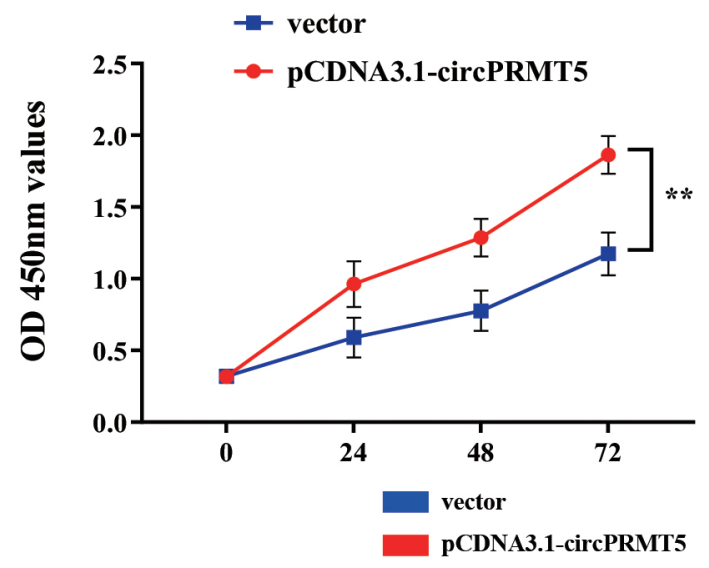

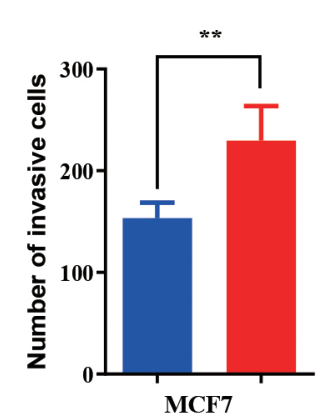

Figure 4: Functional assay of circPRMT5 overexpression on proliferation, invasion, and migration in MCF-7 cell line. A: RT-qPCR method was conducted to detect the overexpression efficiency compared with vector. A: transfection with pcDNA3.1-circPRMT5 can effectively overexpress circPRMT5 in cells, $\mathrm{p}<0.01$. B: CCK-8 method detects the light absorption value of $0 \mathrm{~h}, 24 \mathrm{~h}, 48 \mathrm{~h}$, and $72 \mathrm{~h}$ at $450 \mathrm{~nm}$ wavelength in different groups (vector and pCDNA3.1-circPRMT5) in MCF7 cells. C: Transwell chamber (without matrigel) experiment was used to test the migration ability in different groups (vector and pCDNA3.1-circPRMT5) of MCF7 cells. D: Transwell chamber (adding Matrigel) experiment to detect the invasion ability in different groups (vector and pCDNA3.1-circPRMT5) of MCF7 cells.

paracancerous tissues. The results showed that circPRMT5 expression in $\mathrm{BC}$ tumors was significantly higher than that of paracancerous tissues $(\mathrm{P}<0.001$, Figure $2 \mathrm{~A})$. Consistently, circPRMT5 expression level in multiple $\mathrm{BC}$ cell lines was also significantly increased than that in normal cells $(\mathrm{P}<0.01$, Figure $2 \mathrm{~B})$. In addition, the levels of circPRMT5 collected from serum in BC patients were also elevated when compared with healthy controls ( $\mathrm{P}$ $<0.001$, Figure 2C). Moreover, the receiver operating characteristics (ROC) curve of circPRMT5 expression level was used for predicting the $\mathrm{BC}$ cancer status from $90 \mathrm{BC}$ tumor samples and 90 paracancerous tissues. AUC (area under the curve) $>0.8$ indicates that circPRMT5 expression with a cut-off value of 3.0 could be a good predictor for 
A

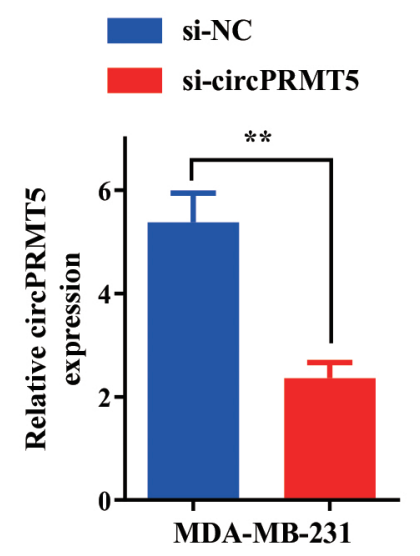

B

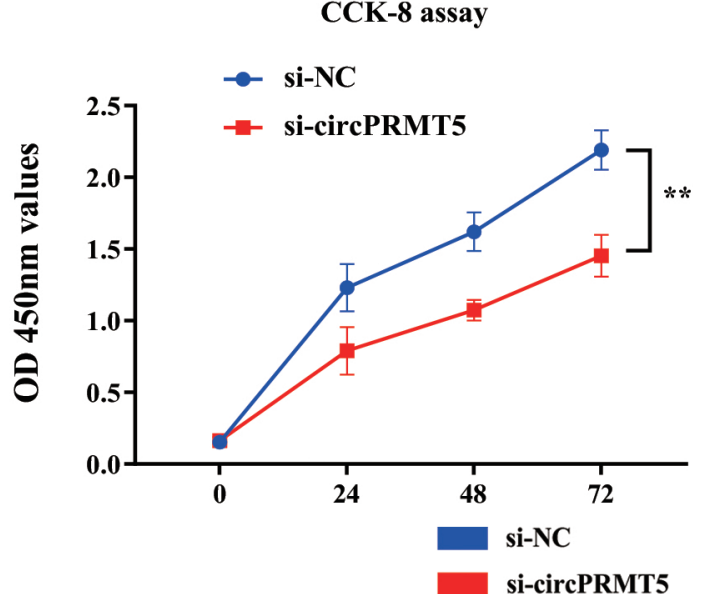

C

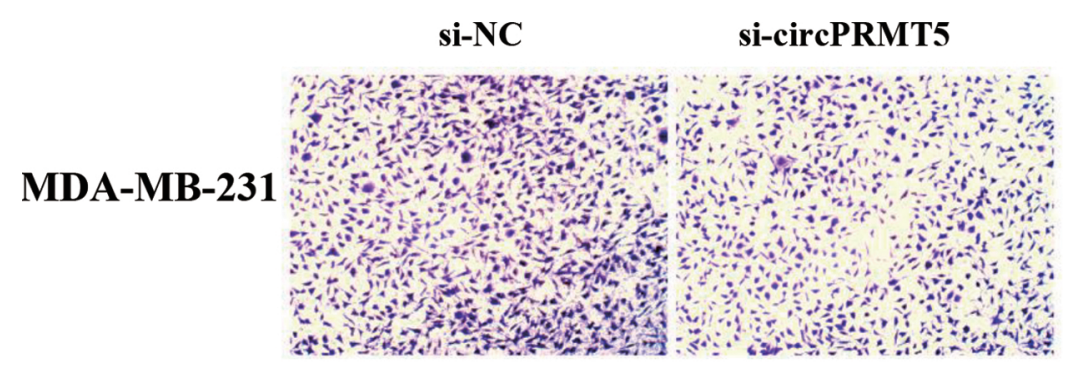

D

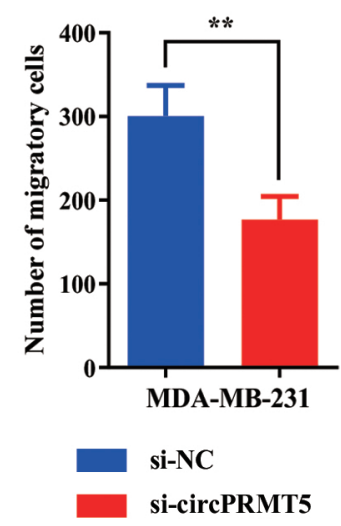

si-NC si-circPRMT5
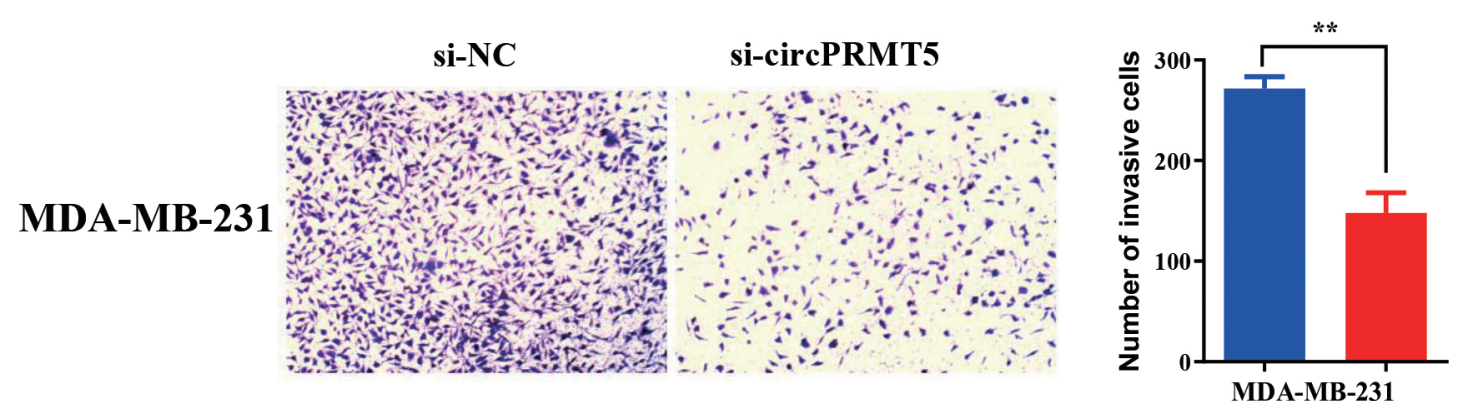

Figure 5: Functional assay of circPRMT5 overexpression on proliferation, invasion, and migration in MDA-MB-231cell line. A: Select the BC cell line with the highest circPRMT5 expression (MDA-MB-231) from the results in Figure 1B for knockdown using si-circPRMT5, and the RT-qPCR method detects knockdown efficiency. B: CCK-8 method detects the light absorption value of $0 \mathrm{~h}, 24 \mathrm{~h}, 48 \mathrm{~h}$, and $72 \mathrm{~h}$ in different groups (si-NC and si-circPRMT5) at 450nm wavelength in MDA-MB-231 cells. C: Transwell chamber (without matrigel) experiment checks the migration ability of different groups (si-NC and si-circPRMT5) of MDA-MB-231 cells. D: Transwell chamber (adding Matrigel) experiment to detect the invasion ability of different groups (si-NC and si-circPRMT5) of MDA-MB-231 cells.

breast cancer with a $78.6 \%$ sensitivity and $91.3 \%$ specificity (Figure 2D). As shown in Table, 90 patients were divided into low and high expression groups based on the median value of circPRMT5 expression, and a high circPRMT5 expression level was associated with tumor maximum diameter, degree of differentiation, TNM staging, lymph node metastasis distant metastasis $(\mathrm{P}<0.05)$. However,
circPRMT5 expression level was not associated with ER, PR, HER2 status ( $\mathrm{P}>0.05)$ (Table).

3.2. Relationship between circPRMT5 and prognosis of BC

To examine whether circPRMT5 expression level is correlated with the prognosis of BC patients, we divided the 90 patients into low expression group $(n=45)$ and 
high expression group $(n=45)$ based on the median value of circPRMT5 expression detected by qPCR in Figure 1A. From the Kaplan-Meier survival curve, the overall survival time and progression-free survival time of $\mathrm{BC}$ patients in the circPRMT5 high expression group were significantly shorter than that of the low expression group $(\mathrm{P}<0.001)$ (Figure 3$)$. Taken together, the data suggest that circPRMT5 is upregulated in BC and higher circPRMT5 expression correlates with a poor prognosis in $\mathrm{BC}$ patients.

\subsection{Functional analysis of circPRMT5 overexpression} and silencing on proliferation, invasion, and migration in $\mathrm{BC}$ cell line

From the results in Figure 1B, MCF7 cells showed a relatively lower expression level of circPRMT5 than other $\mathrm{BC}$ cell lines. We selected MCF7 cell line for overexpression experiment using plasmid pCDNA3.1-circPRMT5. The functional effects of circPRMT5 in the proliferation, invasion, and migration were analyzed. RT-qPCR analysis showed that transfection of pCDNA3.1-circPRMT5 was capable to increase circPRMT5 expression by more than four times in MCF7 cells (Figure 4A). CCK8 proliferation assay showed that the overexpression of circPRMT5 could promote cell proliferation (Figure 4B). Transwell migration and invasion assays demonstrated that circPRMT5 overexpression enhanced the migration and invasion ability of MCF7 cells (Figure 4C and D). Collectively, these results indicated that elevated circPRMT5 expression promotes cell proliferation, migration, and invasion in $\mathrm{BC}$ cells.

To further confirm an indispensable role of circPRMT5 in BC cells, we knocked down circPRMT5 using siRNA specifically targeting circPRMT5 in MDA-MB-231 cells (Figure 5A). In comparison with the control group, the proliferation of MDA-MB-231 cells with circPRMT5 knockdown was significantly suppressed (Figure 5B). Cell migration and invasion assay further showed that the number of migratory and invasive BC cells were largely reduced after circPRMT5 silencing (Figure $5 \mathrm{C}$ and D). Taken together, these results suggest that circPRMT5 is indispensable for supporting cell proliferation, migration, and invasion in $\mathrm{BC}$ cells.

\section{Discussion}

In the study, we found that circPRMT5 was upregulated in $\mathrm{BC}$ tissue as compared to adjacent normal breast tissue. Consistently, circPRMT5 was also upregulated in serum samples collected from BC patients when compared with healthy controls. Importantly, a higher circPRMT5 expression level correlated with a poor prognosis in $\mathrm{BC}$ patients. The overexpression of circPRMT5 in BC cells promoted the proliferation, invasion, and migration; while the knockdown of circPRMT5 inhibited cell proliferation, invasion, and migration. These data suggest that circPRMT5 functions as an oncogenic factor in BC.

Many risk factors including age, hormone levels, fertility, breastfeeding, and gene mutations have been reported to be responsible for BC occurrence $[19,20]$. The metastasis of the Her2-negative invasive breast cancer cells is linked with poor prognosis [21, 22]. Previous studies have been revealed genetic mutations contributing to $\mathrm{BC}$ predisposition. Mutations or dysregulation of tumorsuppressorgenes, such as BRCA-1, BRCA-2, p53, and PTEN play an important role in the initiation and development of BC [23-25]. Importantly, accumulating evidence uncovers the critical role of noncoding RNA such cicrRNAs in the progression of $\mathrm{BC}$, which may regulate different biological processes by sponging microRNA. For example, the overexpression of circ-ABCB10 and circ_001783 were found to facilitate $\mathrm{BC}$ progression through sponging miR1271 and miR-200c-3p, respectively [26, 27]. In addition, circ_0003645 was shown to promote BC proliferation by targeting miR-139-3p/HMGB1 signaling axis [28], and circ_0001667 could enhance BC cell survival by regulating TAZ in Hippo signal pathway [29]. In our study, we also demonstrated the upregulation of CircPRMT5 in BC tumors and cell lines, and the silencing of CircPRMT5 suppressed the aggressive phenotype of BC cells. We therefore propose that CircPRMT5 may function as an oncogene in $\mathrm{BC}$ development.

CircPRMT5 is one of the circRNAs whose abnormal expression has been documented in a variety of malignant tumor tissues. circPRMT5 is highly expressed in gastric cancer patients, and the upregulation of circPRMT5 can promote the expression of oncogene MYC through sponging miR-145 and miR-1304 [30]. In bladder cancer, overexpressed circPRMT5 regulates the epithelialmesenchymal transition of bladder cancer cells and promotes the metastasis [13]. Moreover, the expression level of circPRMT was also elevated in esophageal cancer cells to augment the migration [31]. In hepatoma and nonsmall cell lung cancer (NSCLC), upregulation of circPRMT5 facilitates cancer progression via targeting HK2 or EZH2 [15,16]. Therefore, it seems that circPRMT5 plays multifaceted roles by targeting different pathways to support tumorigenesis in different cancers.

In line with these previous findings, our present study showed that the expression level of circPRMT5 is significantly increased in human BC tissues, serum samples of $\mathrm{BC}$ patients and $\mathrm{BC}$ cells. High expression of circPRMT5 is associated with a poorer prognosis in $\mathrm{BC}$ patients. These findings are consistent with a recent study in which the upregulation of circPRMT5 in breast cancer cells contributes to the aggressive phenotype by mediating the miR-509-3p/TCF7L2 axis to activate the PI3K/AKT pathway [32]. Apart from that, our study further demonstrated that circPRMT5 is indispensable 
for the invasion and migration capabilities of BC cells. Furthermore, the clinical parameter analysis showed that a high circPRMT5 expression level is correlated with larger tumor size, more advanced TNM stage, poorer differentiation, and more lymph node metastasis distant metastasis. Together, our results and the recent study highlighted a functional role of circPRMT5 in supporting the malignant phenotype in $\mathrm{BC}$ cells. However, but no correlation was observed between circPRMT5 and ER, PR, HER2 status, indicating that the upregulation of circPRMT5 in BC may not result from dysregulated hormone receptor signaling.

To sum up, our study found that circPRMT5 is highly expressed in $\mathrm{BC}$ tumor and the plasma of $\mathrm{BC}$ patients. The higher circPRMT5 expression level is related to more aggressive tumor phenotypes including more advanced tumor staging, lymph node metastasis, and poorer differentiation in tumor. Our data suggest that circPRMT5 might be a potential diagnostic and prognostic biomarker for BC. However, this study is limited to the analysis of clinical samples and the functional assays in cell

\section{References}

1. Bray F, Ferlay J, Soerjomataram I, Siegel R L, Torre L A et al. Global cancer statistics 2018: GLOBOCAN estimates of incidence and mortality worldwide for 36 cancers in 185 countries. CA: a Cancer Journal for Clinicians 2018; 68 (6): 394-424. doi: 10.3322/caac. 21492

2. Carioli G, Bertuccio P, Malvezzi M, Rodriguez T, Levi F et al. Cancer mortality predictions for 2019 in Latin America. International Journal of Cancer 2020; 147 (3): 619-632. doi: 10.1002/ijc.32749

3. Li J, Cai H, Sun H, Qu J, Zhao B et al. Extracts of Cordyceps sinensis inhibit breast cancer growth through promoting M1 macrophage polarization via NF-kappaB pathway activation. Journal of Ethnopharmacology 2020; 260: 112969. doi: 10.1016/j.jep.2020.112969

4. Li Y, Qin J, Wu J, Dai X, Xu J. High expression of OSR1 as a predictive biomarker for poor prognosis and lymph node metastasis in breast cancer. Breast Cancer Research and Treatment 2020; 182 (1): 35-46. doi: 10.1007/s10549-02005671-w

5. Wang Y, Ren S, Wang Z, Wang Z, Zhu N et al. Chemokines in bone-metastatic breast cancer: Therapeutic opportunities. International Immunopharmacology 2020; 87: 106815. doi: 10.1016/j.intimp.2020.106815

6. Peng W, Li T, Pi S, Huang L, Liu Y. Suppression of circular RNA circDHCR24 alleviates aortic smooth muscle cell proliferation and migration by targeting miR-149-5p/MMP9 axis. Biochemical and Biophysical Research Communications 2020; 529 (3): 753-759. doi: 10.1016/j.bbrc.2020.06.067 experiments. Further experiments are needed to validate the tumorigenic role of circPRMT5 in BC animal model and to elucidate the molecular targets of circPRMT5.

\section{Acknowledgment}

The study was supported by Tumor Microenvironment and Immunotherapy Hubei Provincial Key Laboratory (Three Gorges University) Open Fund Project (2017KZL07).

\section{Conflict of interest}

There is no conflict of interest related to this study.

\section{Informed consent}

This study was approved by the Institutional Review Board of Second People's Hospital of China Three Gorges University, Yichang Second People's Hospital (LL-2016011). Patient samples were obtained after written informed consent had been obtained, and all procedures were performed in this study involving human participants were in accordance with the Declaration of Helsinki (as revised in 2013).
7. Pan Y, Lou J, Wang H, An N, Chen $\mathrm{H}$ et al. CircBA9.3 supports the survival of leukaemic cells by up-regulating c-ABL1 or BCR-ABL1 protein levels. Blood Cells, Molecules and Diseases 2018; 73: 38-44. doi: 10.1016/j.bcmd.2018.09.002

8. Guo X, Dai X, Liu J, Cheng A, Qin C et al. Circular RNA circREPS2 Acts as a Sponge of miR-558 to Suppress Gastric Cancer Progression by Regulating RUNX3/beta-catenin Signaling. Molecular Therapy Nucleic Acids 2020; 21: 577-591. doi: 10.1016/j.omtn.2020.06.026

9. Zhang J, Wang H, Wu K, Zhan F, Zeng H. Dysregulated circRNA_100876 contributes to proliferation and metastasis of colorectal cancer by targeting microRNA-516b (miR516b). Cancer Biology \& Therapy 2020; 21 (8): 733-740. doi: 10.1080/15384047.2020.1776075

10. Bian L, Zhi X, Ma L, Zhang J, Chen P et al.Hsa_circRNA_103809 regulated the cell proliferation and migration in colorectal cancer via miR-532-3p / FOXO4 axis. Biochemical and Biophysical Research Communications 2018; 505 (2): 346-352. doi: 10.1016/j.bbrc.2018.09.073

11. Ma X, Liu C, Gao C, Li J, Zhuang J et al. circRNA-associated ceRNA network construction reveals the circRNAs involved in the progression and prognosis of breast cancer. Journal of Cellular Physiology 2020; 235 (4): 3973-3983. doi: 10.1002/ jcp.29291

12. Li C, Zhou H. Circular RNA hsa_circRNA_102209 promotes the growth and metastasis of colorectal cancer through miR761-mediated Ras and Rab interactor 1 signaling. Cancer Medicine 2020; 9 (18): 6710-6725. doi: 10.1002/cam4.3332 
13. Chen X, Chen R X, Wei W S, Li Y H, Feng Z H et al. PRMT5 Circular RNA Promotes Metastasis of Urothelial Carcinoma of the Bladder through Sponging miR-30c to Induce EpithelialMesenchymal Transition. Clinical Cancer Research: an Official Journal of The American Association for Cancer Research 2018; 24 (24): 6319-6330. doi: 10.1158/1078-0432.CCR-181270

14. Yang B, Du K, Yang C, Xiang L, Xu Y et al. CircPRMT5 circular RNA promotes proliferation of colorectal cancer through sponging miR-377 to induce E2F3 expression. Journal of Cellular and Molecular Medicine 2020; 24 (6): 3431-3437. doi: 10.1111/jcmm.15019

15. Wang Y, Li Y, He H, Wang F. Circular RNA circ-PRMT5 facilitates non-small cell lung cancer proliferation through upregulating EZH2 via sponging miR-377/382/498. Gene 2019; 720: 144099. doi: 10.1016/j.gene.2019.144099

16. Ding Z, Guo L, Deng Z, Li P. Circ-PRMT5 enhances the proliferation, migration and glycolysis of hepatoma cells by targeting miR-188-5p/HK2 axis. Annals of Hepatology 2020; 19 (3): 269-279. doi: 10.1016/j.aohep.2020.01.002

17. Sun L, Zheng W, Liu Q, Ge L. Valproic Acid Protects Chondrocytes from LPS-Stimulated Damage via Regulating miR-302d-3p/ITGB4 Axis and Mediating the PI3K-AKT Signaling Pathway. Frontiers in Molecular Biosciences 2021; 8: 633315. doi: 10.3389/fmolb.2021.633315

18. Yu Y, Xiao C, Tan L, Wang Q, Li X et al. Cancer-associated fibroblasts induce epithelial-mesenchymal transition of breast cancer cells through paracrine TGF-beta signalling. British Journal of Cancer 2014; 110 (3): 724-32. doi: 10.1038/ bjc. 2013.768

19. Sung H, Devi B, Tang T, Rosenberg P, Anderson W et al. Divergent breast cancer incidence trends by hormone receptor status in the state of Sarawak, Malaysia. International Journal of Cancer 2020; 147 (3): 829-837. doi: 10.1002/ijc.32812

20. Lienhoop T, Smetko M, Green L. Breast cancer in transgender women: A case report. Clinical Imaging 2020; 68: 20-23. doi: 10.1016/j.clinimag.2020.05.006

21. Rojas K, Manasseh D, Rojas M, Mattocks A, Portnow L et al. The natural history of untreated estrogen receptor-positive, Her2-negative invasive breast cancer. Breast Cancer Research and Treatment 2020; 182 (1): 79-83. doi: 10.1007/s10549-02005666-7

22. Miyashita H, Cruz C, Malamud S. Risk factors for skeletalrelated events in patients with bone metastasis from breast cancer undergoing treatment with zoledronate. Breast Cancer Research and Treatment 2020; 182 (2): 381-388. doi: 10.1007/ s10549-020-05712-4
23. Cragun D, Weidner A, Tezak A, Clouse K, Pal T. Cancer risk management among female BRCA1/2, PALB2, CHEK2, and ATM carriers. Breast Cancer Research and Treatment 2020; 182 (2): 421-428. doi: 10.1007/s10549-020-05699-y

24. Xian J, Cheng Y, Qin X, Cao Y, Luo Y et al. Progress in the research of p53 tumour suppressor activity controlled by Numb in triple-negative breast cancer. Journal of Cellular and Molecular Medicine 2020; 24 (13): 7451-7459. doi: 10.1111/ jcmm.15366

25. Brunner A, Suryo Rahmanto A, Johansson H, Franco M, Viiliainen $J$ et al. PTEN and DNA-PK determine sensitivity and recovery in response to WEE1 inhibition in human breast cancer. Elife 2020; 9. doi: 10.7554/eLife.57894

26. Liu Z, Zhou Y, Liang G, Ling Y, Tan W et al. Circular RNA hsa_circ_001783 regulates breast cancer progression via sponging miR-200c-3p. Cell Death \& Disease 2019; 10 (2): 55. doi: 10.1038/s41419-018-1287-1

27. Liang H, Zhang X, Liu B, Jia G, Li W. Circular RNA circ$\mathrm{ABCB} 10$ promotes breast cancer proliferation and progression through sponging miR-1271. American Journal of Cancer Research 2017; 7 (7): 1566-1576.

28. Zhang J, Ke S, Zheng W, Zhu Z, Wu Y. Hsa_circ_0003645 Promotes Breast Cancer Progression by Regulating miR-1393p/HMGB1 Axis. Oncotargets and Therapy 2020; 13: 1036110372. doi: 10.2147/OTT.S265796

29. Geng Z, Wang W, Chen H, Mao J, Li Z et al. Circ_0001667 promotes breast cancer cell proliferation and survival via Hippo signal pathway by regulating TAZ. Cell \& Bioscience 2019; 9: 104. doi: 10.1186/s13578-019-0359-y

30. Du W, Li D, Guo X, Li P, Li X et al. Circ-PRMT5 promotes gastric cancer progression by sponging miR-145 and miR1304 to upregulate MYC. Artificial Cells Nanomedicine and Biotechnology 2019; 47 (1): 4120-4130. doi: 10.1080/21691401.2019.1671857

31. Zhang L, Wang B, Yang J, Yang H. Circ-PRMT5 stimulates migration in esophageal cancer by binding miR-203. European Review for Medical and Pharmacological Sciences 2020; 24 (19): 9965-9972. doi: 10.26355/eurrev_202010_23209

32. Wu D, Jia H, Zhang Z, Li S. Circ-PRMT5 promotes breast cancer by the miR-509-3p/TCF7L2 axis activating the PI3K/ AKT pathway. The Journal of Gene Medicine 2021; 23 (2): e3300. doi: 10.1002/jgm.3300 